\title{
Evaluation of Auto-Regressive Modeling Procedures for the Detection of Abnormal Intra-QRS Potentials Using a Boundary Element Electrocardiogram Model
}

\author{
MC Svendsen ${ }^{1}$, TF Oostendorp ${ }^{2}$, EJ Berbari $^{1}$ \\ ${ }^{1}$ Department of Biomedical Engineering, IUPUI, Indianapolis, Indiana, USA \\ ${ }^{2}$ Department of Cognitive Neuroscience, University Medical Center St Radboud, Nijmegen, the \\ Netherlands
}

\begin{abstract}
Auto-regressive modeling procedures $(A R)$ have been used to quantify low magnitude components of abnormal activation within the QRS complex, called abnormal intra-QRS potentials (AIQPS). Research is still needed to identify the range in which the AR model can detect $A I Q P$ sources in different locations in the heart.

A ventricular source model and forward model of electrocardiography called ECGSIM was used to test the ability of the AR model in detecting delays at various locations in the ventricles. A high resolution ECGSIM heart model was created to provide greater temporal and spatial control of the AIQP sources.

The overall optimal model orders in this study were at an intermediate range seen previously in the literature. The ability of the AR model to detect the AIQP sources was dependent on the resolution of the heart model and the size and location of the AIQP sources.
\end{abstract}

\section{Introduction}

Signal averaged electrocardiograms (SAECGs) have been used to identify patients at high risk for ventricular arrhythmias using a marker called abnormal intra-QRS potentials (AIQPs) [1]. AIQPs are low magnitude, abnormal components of disrupted or delayed ventricular activation within the QRS complex, which occur in the border zone regions around myocardial infarctions [2]. The identification of AIQPs is achieved through autoregressive modeling procedures (AR). The QRS complex is assumed to consist of a normal component of activation, which is entirely predictable and smooth, and an abnormal component of activation, which is unpredictable and transient. The abnormal component of activation is the residual and is seen as a measure of the AIQPs. Studies show that AIQPs are useful in detecting transiently induced myocardial ischemia and have a predictive value close to the currently used standards for arrhythmia prediction, like QRS duration (QRSD) [2,3]. However, due to a lack of knowledge about the AIQP locations in many of these studies, questions still remain about the range of the AR model in detecting delays at different locations in the ventricles.

\section{Methods}

\subsection{Cardiac source and forward model}

A well established ventricular source model and forward model of electrocardiography, called ECGSIM, was used in this study to test the range in which the AR model could detect delays in ventricular activation [4]. The ECGSIM ventricular source model is based on experimental data, the equivalent surface source model, and the bidomain model of the heart [4]. A preexisting low resolution ECGSIM heart model with 576 nodes was utilized to create a high resolution ECGSIM heart model with 5,176 nodes. The location and depolarization times for the additional nodes in the high resolution ECGSIM heart model were derived using the interpolation methods described by Oostendorp et al [5]. The interpolation methods were verified using the depolarization isochrone maps and the QRS complexes for normal ventricular activation, which are seen in Figures 1 and 2.

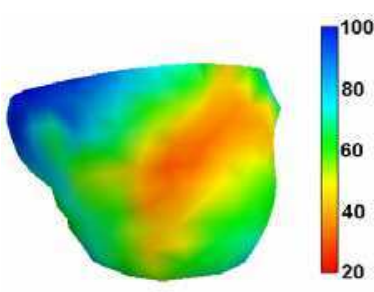

(a)

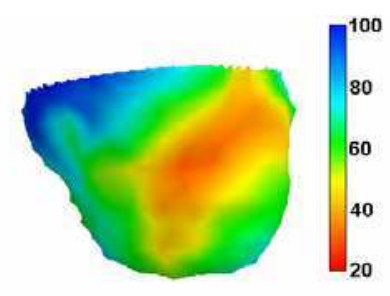

(b)
Figure 1: Depolarization isochrones on the ventricular anterior surface for (a) the low resolution ECGSIM heart model and (b) the high resolution ECGSIM heart model. 

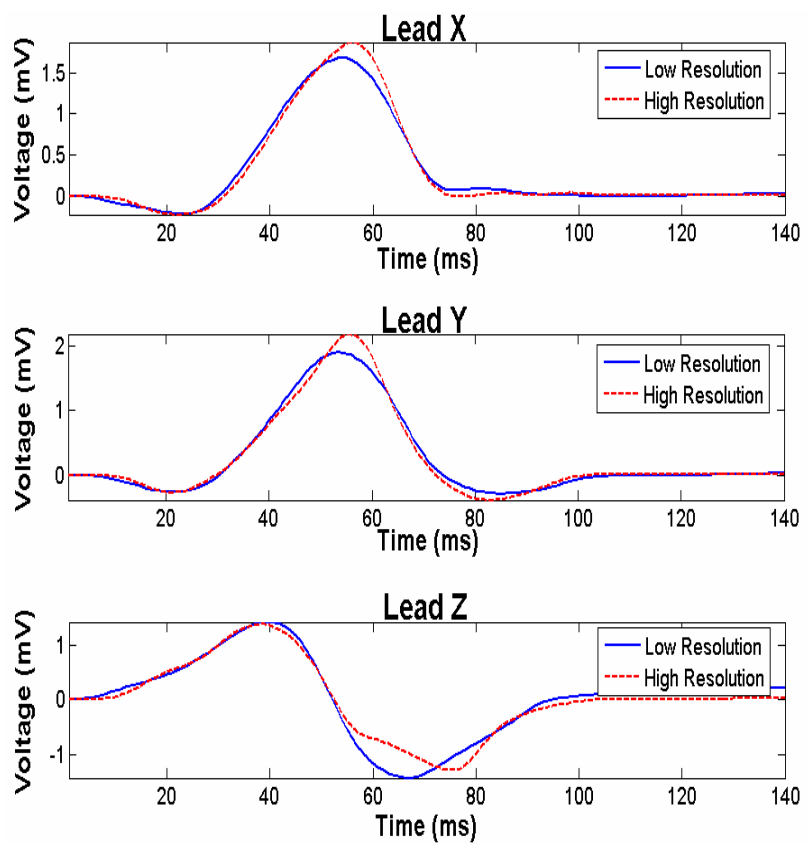

Figure 2: QRS complexes for normal ventricular activation in the $\mathrm{X}, \mathrm{Y}$, and $\mathrm{Z}$ leads using the low and high resolution ECGSIM heart models.

The low and high resolution ECGSIM heart models were used to generate a series of normal and abnormal QRS complexes. Abnormal QRS complexes contained delays in depolarization timing, which ranged from $2 \mathrm{~ms}$ to $50 \mathrm{~ms}$. These delays were made at six different locations in the ventricles, which included sections of the LV and RV epicardial surfaces and the RV septal wall. At each ventricular location, delays were made to a single node and a group of nodes within a $1.0 \mathrm{~cm}, 2.5 \mathrm{~cm}$, and $5.0 \mathrm{~cm}$ radius of the central node. The delays were made along the heart surface and through the entire depth of the myocardium at each location.

\subsection{AR model}

The established AR modeling procedures described by Gomis et al were used to model the normal component of ventricular activation in the QRS complexes generated by the ECGSIM heart models [3]. An impulse response AR model (ARX) was used in the Gomis et al study and this study [3]. The ARX modeled QRS complexes were obtained by passing the input QRS complexes through a discrete cosine transformation, the ARX model, and an inverse discrete cosine transformation. The number of auto-regressive parameters (na) and moving average parameters (nb) determined the fit of the ARX model. ARX modeled QRS complexes were obtained for each case using every combination from na $=5, \mathrm{nb}=5$ to $\mathrm{na}=22, \mathrm{nb}=22$. The residuals were obtained for each case by subtracting the input QRS complex from the ARX modeled QRS complex. The root mean square of the residual was calculated for the duration of the QRS complex and was used as a measure of the abnormal intra-QRS potentials (MAIQPs).

\section{Results}

\subsection{Model order selection}

The optimal model orders were determined for the low and high resolution ECGSIM heart models by finding the model orders which maximized the ratio between the MAIQP for the abnormal case and the MAIQP for the normal case $[3,6]$. The optimal model order was determined for both heart models on a case by case basis for all three leads. In addition, the overall optimal model order was determined for each lead based on the parameters which maximized the number of accurately identified abnormal QRS complexes in all cases. The overall optimal model orders are shown for each lead in both ECGSIM heart models in Table 1.

\begin{tabular}{|c|c|c|c|}
\hline Lead & ECGSIM Model & na & nb \\
\hline \multirow{2}{*}{$\mathrm{X}$} & LRHM & 7 & 17 \\
\cline { 2 - 4 } & HRHM & 8 & 18 \\
\hline \multirow{2}{*}{$\mathrm{Y}$} & LRHM & 9 & 20 \\
\cline { 2 - 4 } & HRHM & 14 & 16 \\
\hline \multirow{2}{*}{$\mathrm{Z}$} & LRHM & 14 & 17 \\
\cline { 2 - 4 } & HRHM & 12 & 22 \\
\hline
\end{tabular}

Table 1: Overall optimal model orders for each lead for the ECGSIM heart models. Abbreviations: LRHM, low resolution heart model and HRHM, high resolution heart model.

\subsection{Range of AIQP detection}

The MAIQP values were used to determine the ability of the ARX model in detecting the AIQP sources for each case. Past studies have provided MAIQP thresholds that delineated the low and high arrhythmia risk patient sets $[3,6]$. Table 2 shows the thresholds from the Gomis et al and Lander et al studies $[3,6]$. In addition, the table shows the MAIQP values for normal ventricular activation using the low and high resolution ECGSIM heart models at the optimal model orders specified by the Gomis et al and Lander et al studies $[3,6]$. The MAIQP values for normal ventricular activation in the low and high resolution ECGSIM heart models were above the Gomis et al threshold for the $\mathrm{Z}$ lead and the Lander et al thresholds for the $\mathrm{X}$ and $\mathrm{Z}$ leads. 
This meant that some of the normal QRS complexes from the ECGSIM heart model would be considered at high risk for arrhythmias using the literature standards $[3,6]$.

In this study, the evaluation the ARX model using MAIQP thresholds from the literature was not appropriate because the thresholds from the literature were determined for specific patient sets at the optimal model orders $[3,6]$. However, a standard was developed from the literature that can be applied to any abnormal case irrespective of the model order selected. This standard, termed the MAIQP threshold ratio, was developed by taking the ratio between the MAIQP threshold value and the MAIQP value for the low arrhythmia risk patient sets using the data from the Gomis et al and the Lander et al studies [3,6]. The MAIQP threshold ratios are shown in Table 2 for the Gomis et al and Lander et al studies [3,6].

In both studies, the threshold ratios are identical for the $\mathrm{Z}$ lead, while differences exist between the threshold ratios in the $\mathrm{X}$ and $\mathrm{Y}$ leads. In fact, the threshold ratio for the $\mathrm{Y}$ lead from the Lander et al study was below one, which indicates the average MAIQP value for the no arrhythmic event patient set was above the MAIQP threshold for that lead [6]. Because the MAIQP threshold ratio was below one in the $\mathrm{Y}$ lead for the Lander et al study, the MAIQP threshold ratios from the Gomis et al study were used in this study $[3,6]$.

\begin{tabular}{|c|c|c|c|}
\hline \multicolumn{4}{|c|}{ MAIQP Comparison Using Gomis et al Study [3] } \\
\hline MAIQP $(\mu \mathrm{V})$ & $\begin{array}{c}\text { Lead } \\
\text { X }\end{array}$ & $\begin{array}{c}\text { Lead } \\
\mathrm{Y}\end{array}$ & $\begin{array}{c}\text { Lead } \\
\text { Z }\end{array}$ \\
\hline Gomis Threshold & 6.5 & 14.5 & 18.9 \\
\hline Gomis Threshold Ratio & 1.25 & 1.08 & 1.20 \\
\hline ECGSIM LRHM & 3.75 & 4.37 & 23.44 \\
\hline ECGSIM HRHM & 4.06 & 7.12 & 37.68 \\
\hline \multicolumn{4}{|c|}{ MAIQP Comparison Using Lander et al Study [6] } \\
\hline MAIQP $(\mu \mathrm{V})$ & $\begin{array}{c}\text { Lead } \\
\text { X }\end{array}$ & $\begin{array}{c}\text { Lead } \\
\text { Y }\end{array}$ & $\begin{array}{c}\text { Lead } \\
\text { Z }\end{array}$ \\
\hline Lander Threshold & 9.85 & 41.60 & 12.00 \\
\hline Lander Threshold Ratio & 1.15 & 0.98 & 1.20 \\
\hline ECGSIM LRHM & 12.89 & 11.67 & 16.13 \\
\hline ECGSIM HRHM & 23.21 & 13.80 & 32.80 \\
\hline
\end{tabular}

Table 2: MAIQP comparisons using Gomis et al study, Lander et al study, and the ECGSIM study $[3,6]$. Abbreviations: LRHM, low resolution heart model and HRHM, high resolution heart model.

The ability of the ARX model to detect the AIQP sources was dependent on the resolution of the ECGSIM heart model and the location of the AIQP sources. For the low resolution ECGSIM heart model, the smallest delay in ventricular activation (a $2 \mathrm{~ms}$ delay in a single node region) was detected by at least one lead for five out of six locations tested. However, for the high resolution ECGSIM heart model, the smallest delay was detected by at least one lead in one out of six locations tested. These results are reasonable because one node in the high resolution ECGSIM heart model represents a smaller region of ventricular tissue compared to the low resolution ECGSIM heart model.

The determination of the range in which the ARX model could detect the AIQP sources was problematic because the detectability of the sources was dependent on its location in the ventricles. In some cases, a delay could be made to a particular region in the ventricles that was undetected by all three leads; while the same delay could be made to another region that was detected by at least one of the three leads. This case is illustrated in Figure 3. Figures 3(a) and 3(b) both show a case where a $50 \mathrm{~ms}$ delay was made to a single node in the ventricles. Figure 3 (a) shows the case when the delay was made to node 123 on the anterior LV epicardial surface; while Figure 3 (b) shows the case when the delay was made to node 147 on the anterior RV epicardial surface. The MAIQP ratio for the node 123 case is below the threshold and the QRS is considered normal, but the MAIQP ratio for the node 147 case is above threshold and the QRS is considered abnormal.

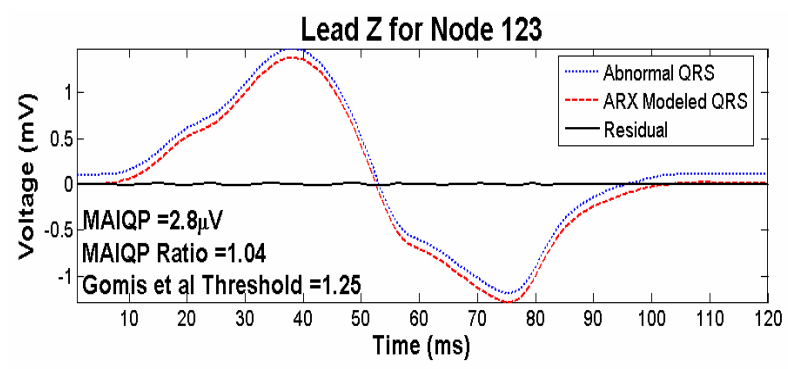

(a)

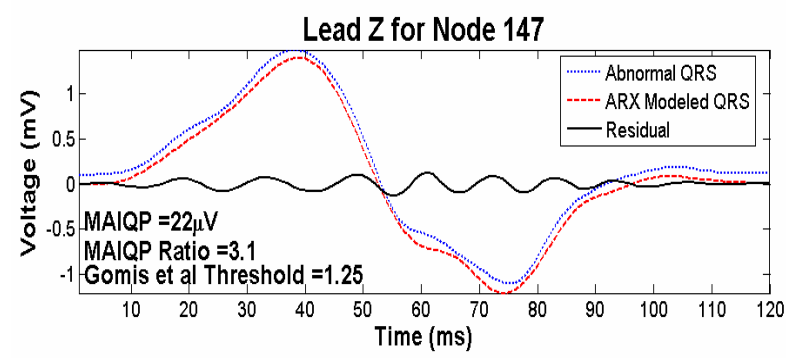

(b)

Figure 3: Abnormal QRS complexes, ARX modeled QRS complexes, and residual for the $\mathrm{Z}$ lead for a $50 \mathrm{~ms}$ delay at (a) node 123 and (b) node 147. 


\section{Discussion and conclusions}

Although the depolarization isochrones shown in Figure 1 are very similar for both ECGSIM heart models, the QRS complexes for normal ventricular activation shown in Figure 2 have some noticeable differences. These differences, which include higher peaks in the $\mathrm{R}$ waves in the $\mathrm{X}$ and $\mathrm{Y}$ leads and a shift and notch in the peak of the $S$ wave in the $Z$ lead, are not unexpected.

In the low and high resolution ECGSIM heart models, a single node represents a specific region of ventricular tissue around the node. The same area that was represented by a single node in the low resolution ECGSIM heart model is now represented by approximately nine times as many nodes in the high resolution ECGSIM heart model. In the high resolution ECGSIM heart model, the interpolation methods caused the newly created nodes to take on depolarization times that were similar, but not identical to the depolarization time of the nearest node from the low resolution ECGSIM heart model.

Certain regions in the low resolution ECGSIM heart model, like the right ventricular outflow tract, contain abrupt geometric changes which cause adjacent triangulations to have nearly opposite normal surface vectors. Because these triangulations share a common node and have opposite normal surface vectors, cancellations will occur between the sources from these triangulations. The increased number of triangulations in the high resolution ECGSIM heart model, the unique depolarization times for the newly created nodes, and the fewer cancellations that occur in the high resolution ECGSIM heart model result in higher peak amplitudes and shifts and notches in the peaks of the QRS complex. In the future when the high resolution ECGSIM heart model is fully optimized, it will provide a more accurate descriptor of ventricular activation compared to the low resolution ECGSIM heart model because of the higher temporal and spatial resolution of the ventricular sources.

In this study, the optimal model orders for the ARX model were at an intermediate level that was similar to the values previously seen in the literature $[3,6]$. Due to the mixture of small and large delay cases, an intermediate set of model orders was optimal for all the cases. The optimal model orders for each case were dependent on many factors that included the temporal size of the delay, the amount of ventricular tissue affected by the delay, the location of the delay in the ventricles, and the resolution of the heart model. Because many of these factors are unknown in clinical settings, a full set of model orders are still necessary to determine the optimal model orders for each QRS complex.

The relationship between the AIQP sources seen in the ECGSIM heart models and actual patient hearts is unknown. However, the results of this study show that the ARX model can detect small delays in activation using simulated QRS complexes from a highly accepted ventricular source model. Although, questions still remain about correlating these findings with actual patient data, the ECGSIM model may be useful in optimizing the ARX model order selection process.

\section{Acknowledgements}

This work was funded by Medtronic Inc. and represents partial fulfillment of a master's of science degree in biomedical engineering for the primary author.

\section{References}

[1] Gomes JA, Winters SL, Stewart D, Horowitz S, Milner M, Barreca P. A new noninvasive index to predict sustained ventricular tachycardia and sudden death in the first year after myocardial infarction: Based on signal-averaged electrocardiogram, radionuclide ejection fraction and Holter monitoring. J Am Coll Cardiol 1987;10:349-57.

[2] Lander P, Gomis P, Warren S, Hartman G, Shuping $\mathrm{K}$, Lazzara R, Wagner G. Abnormal intra-QRS potentials associated with percutaneous transluminal coronary angiography-induced transient myocardial ischemia. J Electrocardiol 2006;39:282-89.

[3] Gomis P, Jones DL, Caminal P, Berbari EJ, Lander P. Analysis of abnormal signals within the QRS complex of the high-resolution electrocardiogram. IEEE Trans on Biomed Eng 1997;44:681-93.

[4] van Oosterom A, Oostendorp TF. ECGSIM: an iteractive tool for studing the genesis of QRST waveforms. Heart 2004;90:165-68.

[5] Oostendorp TF, van Oosterom A, Huiskamp G. Interpolation on a Triangulated 3D Surface. J Comput Phys 1989;80:331-43.

[6] Lander P, Gomis P, Goyal RM, Berbari EJ, Caminal P, Lazzara R, Steinberg JS. Analysis of abnormal intra-QRS potentials: Improved predictive value for arrhythmic events of the signal-averaged electrocardiogram. Circulation 1997;95:1386-93.

Address for correspondence

Edward J. Berbari, Ph.D.

Department of Biomedical Engineering, SL-174

Indiana University Purdue University Indianapolis

723 West Michigan Street

Indianapolis, IN 46202

eberbari@iupui.edu 